Bull. Austral. Math. Soc.

Vol. 40 (1989) [365-369]

\title{
CONSTRUCTING LATTICE-ORDERED FIELDS AND DIVISION RINGS
}

\author{
R.H. REDFIELD
}

\begin{abstract}
Neumann's totally ordered power series fields and division rings may be tipped over to form archimedean lattice-ordered fields and division rings. This process is described and then generalised to produce non-archimedean lattice-ordered fields and division rings in which $1>0$ and, as well, ones in which $1 \ngtr 0$.
\end{abstract}

During the Conference on Ordered Algebraic Structures held in Curaçao in August of 1988, Paul Conrad asked for a general way of constructing examples of lattice-ordered fields and Juhn Dauns asked in a problem session for an example of a proper latticeordered division ring. The present author described to each of them the example described in Section 1 below, in which an archimedean lattice-ordered field or division ring is constructed by "tipping over" a power series field of the kind described by Neumann in [5]. It is an easy matter to generalies the method used in this example to construct more complicated examples in which $1>0$ (Section 2). Furthermore, a modification of the multiplicative structure yields many more examples in which $1 \ngtr 0$ (Section 3).

In what follows, let $\mathbf{F}$ denote a subfield of real numbers.

\section{The EXAMPLE}

Let $\left(\Delta, \cdot, \leq^{\star}\right)$ be a totally ordered group with identity $\imath$. The power series ring $x \prod_{\Delta} \mathbf{F}$ is the set of all elements $z \in \prod_{\Delta} \mathbf{F}$ which have inversely well-ordered support, that is, such that

$$
\operatorname{Supp}(z)=\left\{\delta \in \Delta \mid z_{\delta} \neq 0\right\}
$$

is an inversely well-ordered subset of $\left(\Delta, \leq^{\star}\right)$. These elements are added coordinatewise

$$
(z+w)_{\delta}=z_{\delta}+w_{\delta}
$$

and multiplied as polynomials

$$
(z w)_{\delta}=\sum_{\alpha \beta=\delta} r_{\alpha, \beta} z_{\alpha} w_{\beta}
$$

Received 9 December, 1988

The author presented this material in a talk at Queen's University; he thanks Professor Paulo Ribenboim for a stimulating conversation afterwards.

Copyright Clearance Centre, Inc. Serial-fee code: 0004-9729/89 \$A2.00+0.00. 
where the elements $\left\{r_{\alpha, \beta}\right\} \subseteq \mathbf{F}$ form a factor set in the sense that they satisfy the following conditions:

(i) $r_{\alpha, \beta}>0$ for all $\alpha, \beta \in \Delta$;

(ii) $r_{\alpha \beta, \gamma} r_{\alpha, \beta}=r_{\alpha, \beta \gamma} r_{\beta, \gamma}$ for all $\alpha, \beta, \gamma \in \Delta$;

(iii) $r_{\delta, \imath}=1=r_{2, \delta}$ for all $\delta \in \Delta$.

Note that since the supports are inversely well-ordered, the multiplication is welldefined. The elements of $\Pi_{\Delta} \mathrm{F}$ are then ordered according to the maximal elements in their supports

$$
\begin{gathered}
0<^{\star} z \text { if and only if } 0<z_{\mu} \text { for } \mu=\bigvee \operatorname{Supp}(z) ; \\
w<^{\star} z \text { if and only if } 0<^{\star} z-w .
\end{gathered}
$$

Neumann showed in $[5]$ that the resulting structure $\left(x \mathrm{II}_{\Delta},+, \cdot, \leq^{\star}\right)$, is in general a totally ordered division ring and, if $(\Delta, \cdot)$ is abelian and the factor set satisfies

(iv) $r_{\alpha, \beta}=r_{\beta, \alpha}$ for all $\alpha, \beta \in \Delta$,

a totally ordered field.

Define a new order $\preceq$ on $x \prod_{\Delta} F$ by tipping $\Delta$ over, that is, by letting

$z \preceq w$ if and only if $z_{\delta} \leq w_{\delta}$ for all $\delta \in \Delta$.

This new order is simply the cardinal order on $x \prod_{\Delta} \mathbf{F}$ and hence $\left(x \prod_{\Delta} \mathbf{F},+, \preceq\right)$ is an archimedean lattice-ordered group. From the definition of the multiplication, it is clear that $0 \prec f g$ whenever $0 \prec f$ and $0 \prec g$, and hence that $\left(x \prod_{\Delta} \mathbf{F},+, \cdot, \preceq\right)$ is a lat ticeordered ring. Since $\left(x \prod_{\Delta} \mathbf{F},+, \cdot\right)$ must be a ficld or a division ring, $\left(x \prod_{\Delta} \mathbf{F},+, \cdot, \preceq\right)$ is therefore an archimedean lattice-ordered field or division ring.

\section{The general CONSTRUCtion}

If $(\Delta, \leq)$ is a partially ordered set, then a subset $\Gamma$ of $\Delta$ is locally inversely wellordered if for all $\gamma \in \Gamma,[\gamma, \infty) \cap \Gamma$ is inversely well-ordered; a root system is a partially ordered set $(\Delta, \leq)$ such that for all $\delta \in \Delta,[\delta, \infty)$ is totally ordered; a rooted group is a partially ordered group whose underlying partially ordered set is a root system. We will be interested in orderable partially ordered groups, that is, those partially ordered groups $(\Delta, \cdot, \leq)$ which possess a total order which extends $\leq$ and with respect to which $\Delta$ is a totally ordered group.

It is not hard to find examples of orderable rooted groups. Clearly any totally ordered group is an orderable rooted group, and it is well known that any torsion-free abelian rooted group is orderable (see [2]). To create other orderable rooted groups, 
let $(\Omega, \cdot, \leq)$ be a tolally ordered abelian group with identity $\imath$. Fix a positive integer $n$ and let

$$
\begin{gathered}
\imath<_{n} \omega \text { if and only if } \omega=\xi^{n} \text { for some } \imath<\xi \\
\alpha<_{n} \beta \text { if and only if } \imath<_{n} \alpha^{-1} \beta
\end{gathered}
$$

Then clearly $\left(\Omega, \cdot,<_{n}\right)$ is an orderable rooted group. Furthermore, if $\Omega$ is any orderable rooted group and $\Gamma$ is a trivially ordered orderable group, then it is easy to see that the cardinally ordered product $\Omega \times \Gamma$ is also an orderable rooted group.

Note that for any group $(\Delta, \cdot),\left(2^{\Delta}, \cdot, \subseteq\right)$ is a partially ordered semigroup, where $A B=\{a b \mid a \in A, b \in B\}$ for all $A, B \subset \Delta$.

Now let $(\Delta, \cdot, \leq)$ be an orderable rooted group with identity $\imath$ and suppose that $X$ is a collection of locally inversely well-ordered subsets of $\Delta$ which satisfies the following conditions (see $[6]$ ):
$\left(P_{1}\right) X$ contains all the atoms of $\left(2^{\Delta}, \subseteq\right)$;
$\left(P_{2}\right) \quad X$ is an ideal of the lattice $\left(2^{\Delta}, \subseteq\right)$;
$\left(\mathrm{P}_{3}\right) \quad X$ is a subsemigroup of $\left(2^{\Delta}, \cdot\right)$;
$\left(\mathrm{P}_{4}\right)$ if $A, B \in X$ and $\delta \in \Delta$, then $\{(\alpha, \beta) \in A \times B \mid \alpha \beta=\delta\}$ is finite.

Form the product $\Pi_{\Delta} \mathbf{F}$ and let $x \prod_{\Delta} \mathbf{F}$ denote the set of all elements of $\Pi_{\Delta} \mathbf{F}$ which have their supports in $X$. Define addition and multiplication as in Section 1 above and define a binary relation $\preceq$ on $x \prod_{\Delta} \mathbf{F}$ by letting

$$
\begin{aligned}
& 0 \prec z \text { if and only if } 0<z_{\mu} \text { for all maximal elements } \mu \in \operatorname{Supp}(z) \text {; } \\
& \qquad w \prec z \text { if and only if } 0 \prec z-w .
\end{aligned}
$$

Then $\left(x \Pi_{\Delta} F,+, \cdot, \preceq\right)$ is a lattice-ordered ring (see $\left.[1,3,4,6,7]\right)$.

We wish to choose $X$ so that $\left(x \prod_{\Delta} \mathbf{F},+, \cdot, \preceq\right)$ is a lattice-ordered division ring or a lattice-ordered field. To do this, we reverse the method described in Section 1; we tip $\Delta$ up and use the resulting collection of inversely well-ordered sets as $X$. Specifically, since $\Delta$ is orderable, there exists a total order $\leq^{\star}$ on $\Delta$ which extends $\leq$ and such that $\left(\Delta, \cdot, \leq^{\star}\right)$ is a totally ordered group; let $X$ be the collection of all inversely well-ordered subsets of $\left(\Delta, \leq^{\star}\right)$. Clearly $X$ satisfies $\left(P_{1}\right)-\left(P_{3}\right)$ above; that $X$ satisfies $\left(P_{4}\right)$ is well known (see $[1,5]$ ). To see that $\Gamma \in X$ is locally inversely well-ordered, let $\gamma \in \Gamma$ and let $\Sigma \subseteq\{\delta \in \Gamma \mid \gamma \leqslant \delta\}$. Since $\Sigma \subseteq \Gamma$ and $\Gamma$ is inversely well-ordered in $\left(\Delta, \leq^{\star}\right)$, there exists $\sigma \in \Sigma$ such that $s \leq^{\star} \sigma$ for all $s \in \Sigma$. Then for any $s \in \Sigma, s \not \supset \sigma$ because $\leq^{\star}$ extends $\leq$. However, since $(\Delta, \leq)$ is a root system, $\{\delta \in \Gamma \mid \gamma \leq \delta\}$ is totally ordered, and thus $s \leq \sigma$. We conclude that $\sigma$ is a maximal element of $\Sigma$ in $(\Delta, \leq)$ and hence $\Gamma$ is locally inversely well-ordered in $(\Delta, \leq)$. Therefore, as noted above, $\left(x \prod_{\Delta} \mathbf{F},+, \cdot \preceq\right)$ 
is a lattice-ordered ring. But $\left(x \prod_{\Delta} \mathbf{F},+, \cdot\right)$ is a division ring or a field by [5], and hence $\left(x \prod_{\Delta} \mathbf{F},+, \cdot, \preceq\right)$ is in fact a lattice-ordered division ring or lattice ordered field. Furthermore, if, for each $\alpha \in \Delta$, we let

$$
\hat{\alpha}= \begin{cases}1 & \text { if } \delta=\alpha \\ 0 & \text { otherwise }\end{cases}
$$

then $\hat{\imath}$ is the unit element of $\left(x \prod_{\Delta} F,+, \cdot\right)$ and clearly $0 \prec \hat{\imath}$.

\section{A MODIFICATION OF THE GENERAL METHOD}

By modifying the multiplication but not the addition or the order, the construction described in Section 2 produces latlice-ordered division rings and fields whose unit elements do not exceed zero.

Suppose in general that $(D,+, \cdot)$ is a division ring and that $u$ is a nonzero element in the centre of $D$. Define a binary operator $\nabla_{u}$ on $D$ by letting

$$
x \diamond_{u} u=x y u^{-1} .
$$

Then $\left(D,+, \diamond_{u}\right)$ is also a division ring but with $u$ as unit element.

Now consider the division ring $\left(x \prod_{\Delta} \mathbf{F},+, \cdot, \preceq\right)$ constructed above and suppose that there exists an element $u$ in the centre of $x \prod_{\Delta} F$ such that $0 \nprec u$ but $0 \prec u^{-1}$. Then clearly $0 \prec x \diamond_{u} y$ whenever $0 \prec x$ and $0 \prec y$, and hence $\left(x \prod_{\Delta} \mathbf{F},+, \diamond_{u}, \preceq\right)$ is a lattice-ordered division ring whose unit element does not exceed zero.

Such elements $u$ exist in many of the rings $\left(x \Pi_{\Delta} \mathbf{F},+, \cdot, \preceq\right)$. For suppose that $\Delta$ contains an element $\eta$ in its centre which is not comparable to $\imath$ in $(\Delta, \leq)$ and is such that $r_{\delta, \eta}=r_{\eta, \delta}$ for all $\delta \in \Delta$. Then, since $\left\{r_{\alpha, \beta}\right\} \subseteq \mathbf{F}$ forms a factor set,

$$
r_{\delta, \eta^{-1}}=\left(r_{\delta \eta^{-1}, \eta}\right)^{-1} r_{\delta, \imath} r_{\eta^{-1}, \eta}=\left(r_{\eta, \eta^{-1} \delta}\right)^{-1} r_{\imath, \delta} r_{\eta, \eta^{-1}}=r_{\eta^{-1}, \delta},
$$

and since $\leq^{\star}$ is a total order, either $\eta<^{\star} \imath$ or $\eta^{-1}<^{\star} \imath$. Hence there exists an element $\vartheta$ in the centre of $\Delta$ (either $\vartheta=\eta$ or $\vartheta=\eta^{-1}$ ) which is not comparable to $\imath$ in $(\Delta, \leq$ ), is less than $\imath$ in $(\Delta, \leq)$, and is such that $r_{\delta, \vartheta}=r_{\vartheta, \delta}$ for all $\delta \in \Delta$. Let $u=(\hat{\vartheta}+\hat{\imath})^{-1}$. Then

$$
u_{\delta}= \begin{cases}1 & \text { if } \delta=\vartheta^{2 n} \text { for } n \geq 0 \\ -1 & \text { if } \delta=\vartheta^{2 n+1} \text { for } n \geq 0 \\ 0 & \text { otherwise }\end{cases}
$$

and $u^{-1}=\hat{\vartheta}+\hat{\imath} \succ 0$. If $\vartheta=\vartheta^{1}$ were not a maximal element of $\operatorname{Supp}(u)$ in $(\Delta, \leq)$, then $\vartheta^{m}>\vartheta$ for some $m>1$, and hence, since $\leq^{\star}$ extends $\leq, v^{m}>^{\star} \vartheta$. This contradicts 
our assumption that $\vartheta<\star_{\imath}$, and hence $\vartheta$ must be a maximal element of $\operatorname{Supp}(u)$ in $(\Delta, \leq)$. Thus, since $0>-1=u_{\vartheta}, 0 \nprec u$. To see that $u$ is in the centre of $x \prod_{\Delta} \mathbf{F}$, note that for $z \in x \prod_{\Delta} \mathbf{F}$ and $\delta \in \Delta$,

$$
(z(\hat{\vartheta}+\hat{\imath}))_{\delta}=r_{\delta \vartheta-1, \vartheta} z_{\delta \vartheta-1}+r_{\delta, \imath} z_{\delta}=r_{\vartheta, \vartheta-1} Z_{\vartheta-1 \delta}+r_{\imath, \delta} z_{\delta}=((\hat{\vartheta}+\hat{\imath}) z)_{\delta} .
$$

Thus $u^{-1}$ is in the centre of $x \prod_{\Delta} \mathbf{F}$ and hence so is $u$.

We conclude that whenever $\Delta$ possesses an elment $\vartheta$ in its centre which is not comparable to $\imath$ in $(\Delta, \leq)$ and such that $r_{\delta, \vartheta}=r_{\vartheta, \delta}$ for all $\delta \in \Delta$, there exists a multiplicalion $\vartheta_{u}$ on $x \prod_{\Delta} \mathbf{F}$ such that $\left(x \prod_{\Delta} \mathbf{F},+, \oslash_{u}, \preceq\right)$ is a lattice-ordered division ring whose unit element does not exceed zero. If $\left(x \prod_{\Delta} \mathbf{F},+, \cdot, \preceq\right)$ is a proper latticeordered field, then $(\Delta, \leq)$ clearly posseses such an element and the new multiplication which this element generates is clearly commutative. Therefore, the multiplication on any proper lattice-ordered field $\left(x \prod_{\Delta} \mathbf{F},+, \cdot, \preceq\right)$ can always be modified to produce a lattice-ordered field $\left(x \prod_{\Delta},+, \diamond_{u}, \preceq\right)$ whose unit element does not exceed 0 .

\section{REFERENCES}

[1] Paul Conrad, 'Generalized semigroup rings', J. Indian Math. Soc. 21 (1957), 73-95.

[2] Paul Conrad, Lattice Ordered Groups (Mathematics Departmenl, Tulane University, New Orleans, USA, 1970).

[3] Paul Conrad and Johu Dauns, 'An embedding theorem for lattice-ordered fields', Pacific J. Math. 30 (1969), 385-398.

[4] Paul Conrad, John Harvey and Charles Holland, 'The Hahn embedding theorem for lattice-ordered groups', Trans. Amer. Math. Soc. 108 (1963), 143-169.

[5] B.H. Neumann, 'On ordered division rings', Trans. Amer. Math. Soc. 66 (1949), 202-252.

[0] R.H. Redfield, 'Embeddings into power series rings', submitted.

[7] Paulo Ribenboim, Commutative Convolution Rings of Ordered Monoido (Preprint, Mathematics Department, Queen's University, Kingston, Canada, 1988).

Department of Mathematics and Computer Science

Hamillun College

Clinton NY13323

United States of America 